\title{
Obesity, Circulating Androgens and their Precursors
} Michaela Dušková, Hana Pospíšilová, Martin Hill and Luboslav Stárka*

Institute of Endocrinology, Prague, Czech Republic

\begin{abstract}
Objective: The association of obesity with a lower circulating testosterone level in men is well documented. However, reports on possible changes in the androgen spectrum in obesity are rare.

Methods: To investigate this phenomenon, serum sex hormone-binding globulin (SHBG), testosterone, dihydrotestosterone, androstenedione, dehydroepiandrosterone and its sulphate, 17a-hydroxypregnenolone, 17a-hydroxyprogesterone and gonadotrophins LH and FSH concentrations were measured in fasting blood samples of 224 men divided into three groups - normal (BMI=18-25, n=109, overweight (BMI 25.10-30, n=78) and obese $(\mathrm{BMI}=30.1-39, \mathrm{n}=37)$.
\end{abstract}

Results: A significant decrease in testosterone, dihydrotestosterone, 17a-hydroxypregnenolone, 17a-hydroxyprogesterone and SHBG with increasing body mass index was observed, whereas insignificant changes for dehydroepiandrosterone and its sulphate, androstenedione and gonadotrophins LH and FSH, were found. The ratios of corresponding pairs of steroids were in agreement with the concept that in obesity splitting of the side chain of $\mathrm{C}_{21}$-steroids, and $17 \beta$-hydroxysteroid dehydrogenase-reducing activity are decreased. No changes for steroid $5 \alpha$-reductase or $3 \beta$-hydroxysteroid dehydrogenase (HSD3B2) were found.

Conclusion: The findings demonstrate that, in men with increasing body mass index, the formation of $\mathrm{C}_{19}$ steroids decreases from their $\mathrm{C}_{21}$ precursors and lower $17 \beta$-hydroxysteroid dehydrogenase further confines the production of testosterone and dihydrotestosterone.

Keywords: Obesity; Testosterone; Dihydrotestosterone; Androgens; 17a-hydroxyprogesterone; 17a-hydroxypregnenolone

\section{Introduction}

Reduced testosterone levels, well into the hypogonadal range, are common in male obesity [1-5]. The mechanism of circulating total testosterone concentration decrease is explained by a high expression of aromatase, the enzyme that converts testosterone to estradiol, in adipose tissue and by the resulting elevated estradiol. Together with the increased leptin and adipokines from fat tissue, this triggers inhibition of the hypothalamic-pituitary-gonadal axis $[6,7]$. This results in hypogonadotrophic hypogonadism, which is observed in a large percentage of obese men.

Whereas a handful of publications deal with the relation of testosterone, dihydrotestosterone or estradiol levels to obesity, less attention has been paid to the influence of obesity on androgen metabolism. Some important data were acquired by measuring the intra-adipose metabolism of androgens [8-10]. The activity of enzymes involved in androgen metabolism varies in the different parts of fat tissue and, together with local glucocorticoid activity, constitutes an important factor for fat distribution.

Data concerning circulating androgens and their precursors in obese men, with the exception of that on testosterone or dihydrotestosterone [11], are scarce [12-15]. This short study aims to determine the impact of obesity on the pattern of circulating androgens and to show whether all changes in the concentration of androgens and their precursors 17a-hydroxypregnenolone and 17a-hydroxyprogesterone are proportional to the reduced level of testosterone and if this decline also applies to androgens of mainly adrenal origin.

\section{Materials and Methods}

\section{Subjects}

A group of 224 healthy (except for their obesity and associated symptoms) men aged 20 to 78 with a broad range of body mass index (BMI) 18 to 39 was enrolled in this study. Anthropometric parameters (i.e. weight, height, BMI) were measured. Blood withdrawal was carried out in fasting subjects in the morning between 7:30 and 8:30 a.m. from the forearm vein. Serum was stored at $-80^{\circ} \mathrm{C}$ until it was processed in the laboratory.

The Ethical Committee approved the study and all patients signed informed consent forms before taking part in the study.

\section{Anthropometric data}

Anthropometric data were obtained in a fasting state. Body weight and height were measured in all participants in order to calculate body mass index (BMI). Weight (to the nearest $0.1 \mathrm{~kg}$ ) and height (to the nearest $\mathrm{cm}$ ) were measured. Body mass index was calculated as the weight $(\mathrm{kg})$ divided by height squared $\left(\mathrm{m}^{2}\right)$.

The group of 224 healthy men was divided into three subgroups according to BMI. The first subgroup consisted of 109 men with BMI between 18 and 25 . The second group included 78 men with BMI between 25 and 30 . The third subgroup had 37 men with BMI 30 to 39 .

\section{Steroid analysis}

Laboratory analyses of sex hormone binding globulin (SHBG),

*Corresponding author: Luboslav Stárka, Institute of Endocrinology, Narodni 8 , 11694 Prague 10, Czech Republic, E-mail: Istarka@endo.cz

Received February 21, 2013; Accepted April 09, 2013; Published April 27, 2013

Citation: Dušková M, Pospišilová H, Hill M, Stárka L (2013) Obesity, Circulating Androgens and their Precursors. J Steroids Horm Sci 4: 119. doi:10.4172/21577536.1000119

Copyright: @ 2013 Dušková M, et al. This is an open-access article distributed under the terms of the Creative Commons Attribution License, which permits unrestricted use, distribution, and reproduction in any medium, provided the original author and source are credited. 
Citation: Dušková M, Pospíšilová H, Hill M, Stárka L (2013) Obesity, Circulating Androgens and their Precursors. J Steroids Horm Sci 4: 119. doi:10.4172/2157-7536.1000119

Page 2 of 5

LH, FSH and steroid hormones: dihydrotestosterone, testosterone, 17a-hydroxyprogesterone, dehydroepiandrosterone (DHEA), dehydroepiandrosterone sulfate (DHEAS), 4-androstene-3,17-dione (androstenedione), and 17a-hydroxypregnenolone were carried out.

Serum testosterone was determined by standard radioimmunoassay (RIA) using antiserum anti-testosterone-3-carboxymethyloxim: BSA and testosterone-3-carboxymethyloxim-tyrosylmethyl-ester-[ $\left.{ }^{125} \mathrm{I}\right]$ as a tracer. Intra-assay and inter-assay coefficient variants were $7.2 \%$ and
$10 \%$, respectively, and sensitivity was $0.21 \mathrm{nmol} / \mathrm{l}$. Androstenedione was determined by standard RIA with antiserum anti-androstenedione6-carboxy-methyloxim: BSA and $\left[{ }^{3} \mathrm{H}\right]$ androstenedione as tracer. Intra-assay and inter-assay coefficient variants were $8.1 \%$ and $10.2 \%$ and sensitivity was $0.39 \mathrm{nmol} / \mathrm{l}$. Sexual hormones binding globulin was assayed using an IRMA kit (Orion, Espoo, Finland). Commercial kits (Immunotech, Marseilles, France) were used for the determination of LH, FSH (IRMA kit), 17a-hydroxyprogesterone, dehydroepiandrosterone (DHEA) and dehydroepiandrosterone

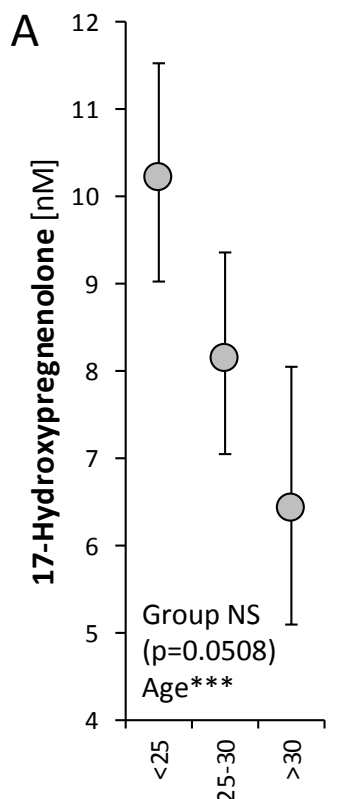

BMI $\left[\mathrm{kg} / \mathrm{m}^{2}\right]$

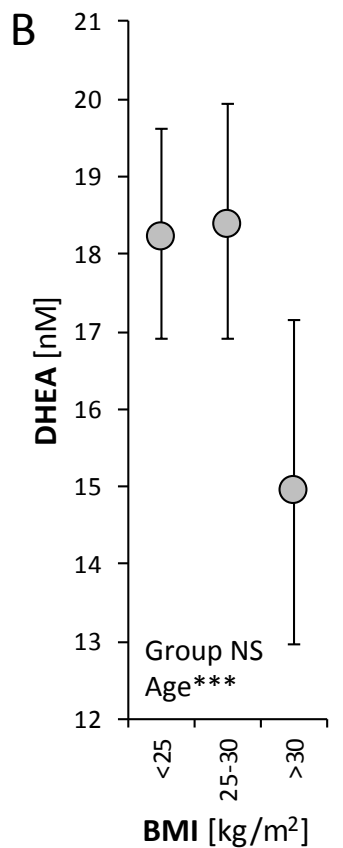

BMI $\left[\mathrm{kg} / \mathrm{m}^{2}\right]$
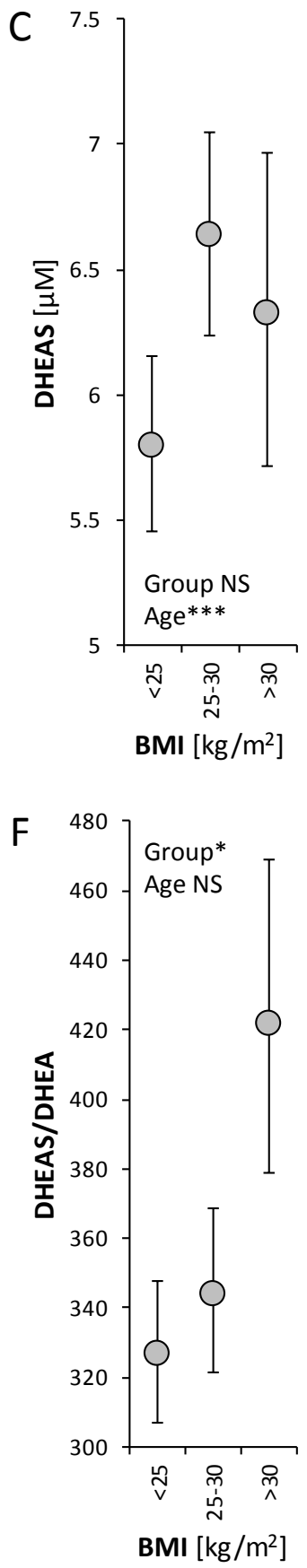
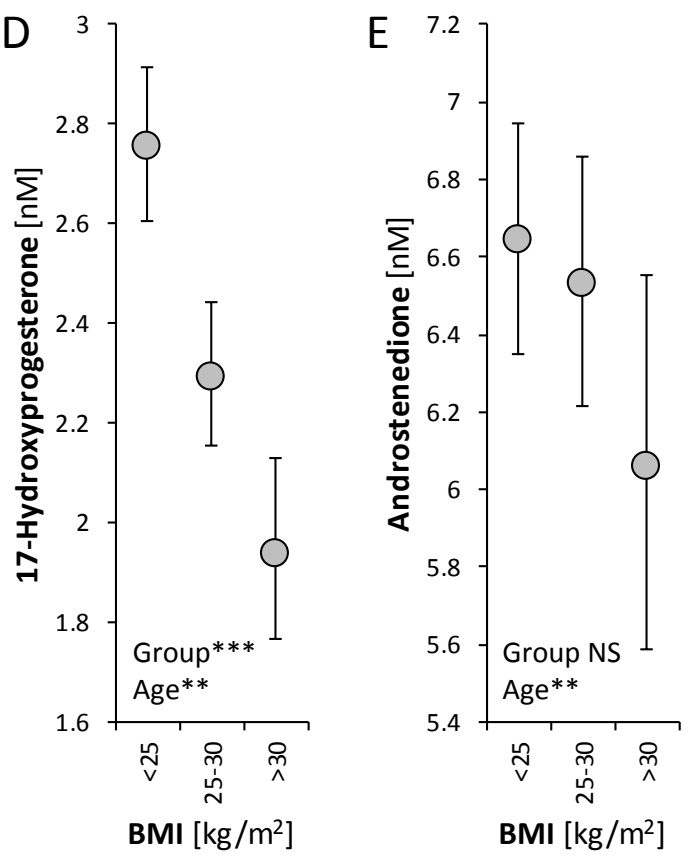

Figure 1: Relationships between steroid levels, DHEAS/DHEA ratio and BMI as evaluated using ANCOVA with BMI group as a main factor and age of the subject as a covariate (for details see statistical data analysis). The circles with error bars represent the group means with their $95 \%$ confidence intervals as computed for least significant difference (LSD) multiple comparisons $(p=0.05)$. The confidence values, which do not overlap, represent statistical significant difference between the corresponding groups. Statistical significance: NS=not significant, ${ }^{*} p<0.05,{ }^{* *} p<0.01,{ }^{* * *} p<0.001$. 

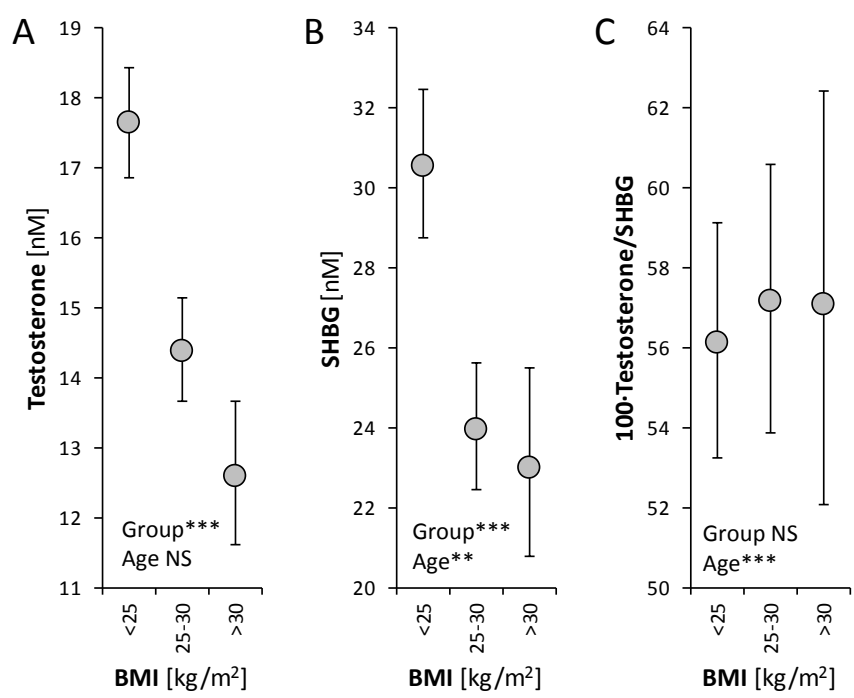

Figure 2: Relationships between levels of testosterone, SHBG, free androgen index (100 testosterone/SHBG), and BMI as evaluated using ANCOVA with $\mathrm{BMI}$ group as a main factor and age of the subject as a covariate (for details see statistical data analysis). The circles with error bars represent the group means with their $95 \%$ confidence intervals as computed for least significant difference (LSD) multiple comparisons $(p=0.05)$. The confidence values, which do not overlap, represent statistical significant difference between the corresponding groups. Statistical significance: NS=not significant, ${ }^{* *} p<0.01$ ${ }^{* * *} p<0.001$.
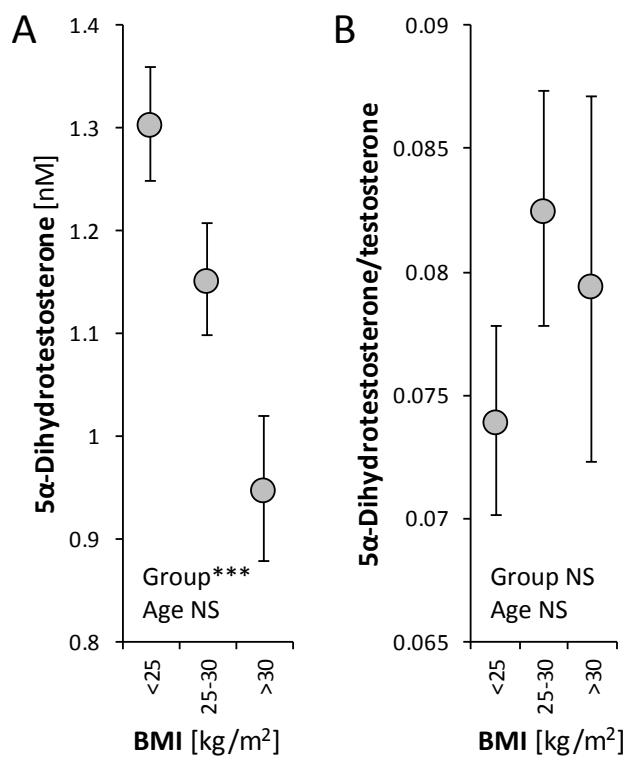

Figure 3: Relationships between levels of $5 \alpha$-dihydrotestosterone, $5 \alpha$-dihydrotestosterone/testosterone ratio and $\mathrm{BMI}$ as evaluated using ANCOVA with BMI group as a main factor and age of the subject as a covariate (for details see statistical data analysis). The circles with error bars represent the group means with their $95 \%$ confidence intervals as computed for leas significant difference (LSD) multiple comparisons $(p=0.05)$. The confidence values, which do not overlap, represent statistical significant difference between the corresponding groups. Statistical significance: NS=not significant, ${ }^{* * *} p<0.001$.

sulfate (DHEAS) (RIA kit). Dihydrotestosterone was determined by an original methodology [16,17]. 17a-Hydroxypregnenolone was determined by an in-house RIA method.

\section{Statistical data analysis}

To evaluate the relationships between dependent variables, we used the ANCOVA model with BMI group as a main factor and age of the subject as a covariate (age-adjusted ANOVA) followed by least significant difference (LSD) multiple comparisons. The original dependent variables and the covariate were transformed by power transformations to attain a constant variance and symmetric distribution of the data and residuals [18]. Statistical software Statgraphic Centurion version XVI (Herndon, VA, USA) was used for the calculations. The homogeneity of the data and residual were checked as described elsewhere [19].

\section{Results}

The comparison of men with normal body mass index, overweight and obese men showed that a significant continuous decrease of parameters for testosterone (Figure 2A), dihydrotestosterone (Figure $3 \mathrm{~A}$ ) and SHBG (Figure 2B) correlates with increasing body mass. Also the circulating both $\mathrm{C}_{21}$ androgen precursors, 17a-hydroxyprogesterone and 17a-hydroxypregnenolone, decrease with increasing BMI (Figures $1 \mathrm{~A}$ and $1 \mathrm{D})$, whereas the changes in androstenedione, DHEA, and DHEAS levels as well as in gonadotrophins do not reach statistical significance (Figures $1 \mathrm{~B}, 1 \mathrm{C}$ and $1 \mathrm{E}$ ). Since the decrease of SHBG (Figure 2B) parallels the decrease of testosterone level (Figure 2A), thus compensating the loss of free testosterone, no change was observed in the free androgen index (Figure 2C).

The dihydrotestosterone : testosterone ratio does not correlate with the degree of obesity (Figure 3B), to the 17a-hydroxyprogesterone : 17a-hydroxpregnenolone ratio or
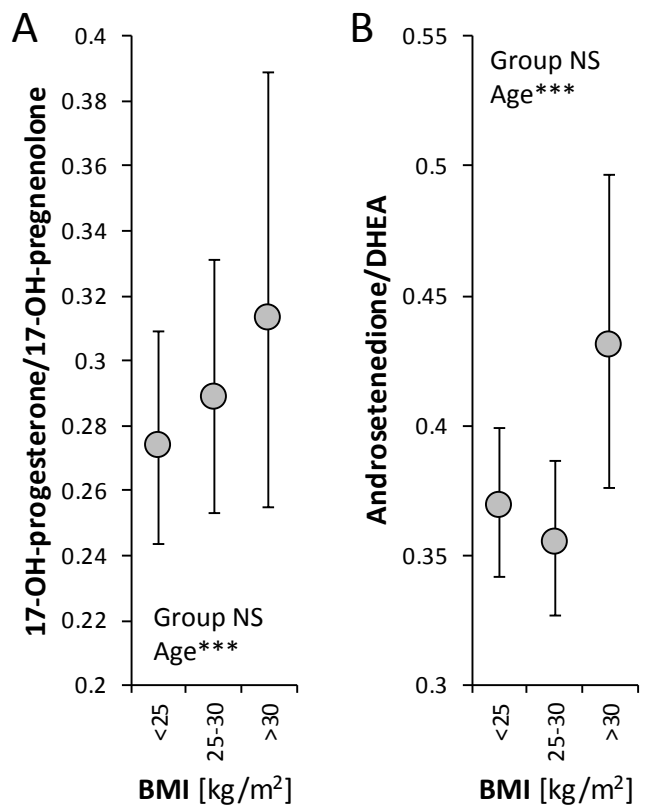

Figure 4: Relationships between steroid ratios reflecting the activity of type $23 \beta$-hydroxysteroid dehydrogenase (HSD3B2) and BMI as evaluated using ANCOVA with BMI group as a main factor and age of the subject as a covariate (for details see statistical data analysis). The circles with error bars represent the group means with their $95 \%$ confidence intervals as computed for least significant difference (LSD) multiple comparisons $(p=0.05)$. The confidence values, which do not overlap, represent statistical significant difference between the corresponding groups. Statistical significance: NS=not significant, ${ }^{* * *} p<0.001$. 
Citation: Dušková M, Pospíšilová H, Hill M, Stárka L (2013) Obesity, Circulating Androgens and their Precursors. J Steroids Horm Sci 4: 119. doi:10.4172/2157-7536.1000119

androstenedione : dehydroepiandrosterone ratio (Figures 4A and 4B), which demonstrates the undisturbed activity of $3 \beta$-hydroxysteroid dehydrogenase type 2 (HSD3B2). On the contrary, it is evident from the DHEA : 17 $\alpha$-hydroxypregnenolone and androstenedione : $17 \alpha$-hydroxprogesterone ratios (Figure $5 \mathrm{~A}$ ) that the activity of $\mathrm{C}_{17}, \mathrm{C}_{20}$ lyase (CYP17A1) decreases with the degree of obesity, especially for the $\Delta^{4}$ pathway (Figure 5B).

The testosterone: androstenedione ratio (Figure 6) decreases significantly, which is in agreement with the decreased activity of 17 $\beta$-hydroxysteroid dehydrogenase type 3 (HSD17B3).

Multivariate statistical analysis showed that age was a significant factor for the correlation of BMI and the levels of 17a-hydroxypregnenolone, 17a-hydroxyprogesterone, androstenedione, dehydroepiandrosterone and its sulfate and SHBG. The decrease of testosterone and dihydrotestosterone with increasing BMI was independent of age.

\section{Discussion}

We can derive from the data that testosterone biosynthesis in overweight and obese men is inhibited already in the step of splitting the side chain of $\mathrm{C}_{21}$ steroids and further by the decrease of $17 \beta$-hydroxysteroid dehydrogenase type 3 (HSD17B3) reducing activity. The changed ratio of dehydroepiandrosterone sulfate to DHEA, which might be a consequence of decreased sulfatase or increased sulfotransferase or increased secretion of DHEA sulfate from the adrenals, is of interest.

No changes with increasing BMI were observed with regards to
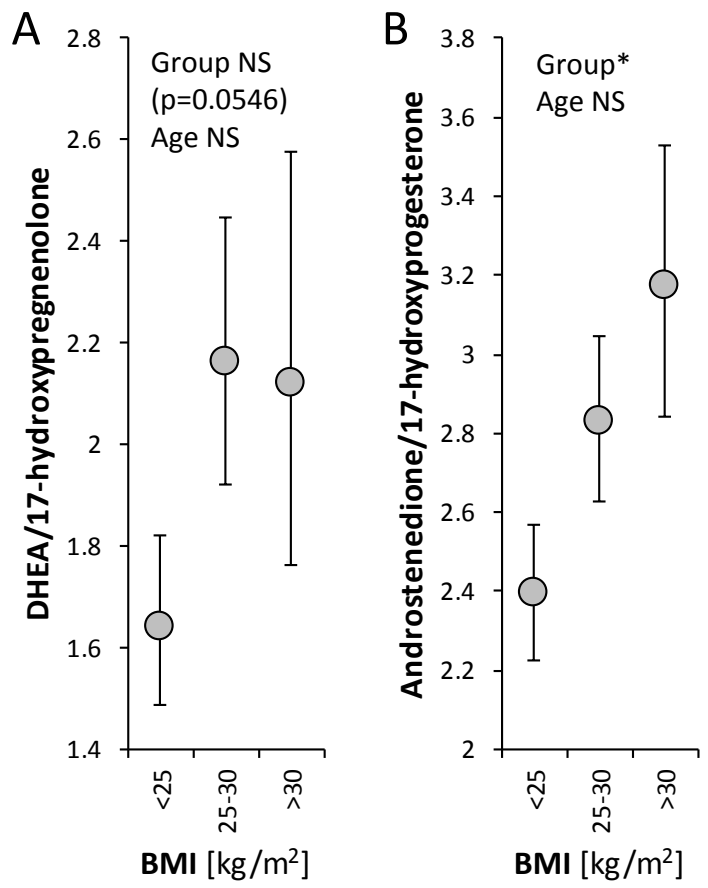

Figure 5: Relationships between steroid ratios reflecting the activity of $\mathrm{C} 17$ hydroxylase, C17,20-lyase (CYP17A1) and BMl as evaluated using ANCOVA with BMI group as a main factor and age of the subject as a covariate (for details see statistical data analysis). The circles with error bars represent the group means with their $95 \%$ confidence intervals as computed for least significant difference (LSD) multiple comparisons $(p=0.05)$. The confidence, which do not overlap represent statistical significant difference between the corresponding groups. Statistical significance: NS=not significant, ${ }^{*} p<0.05$.

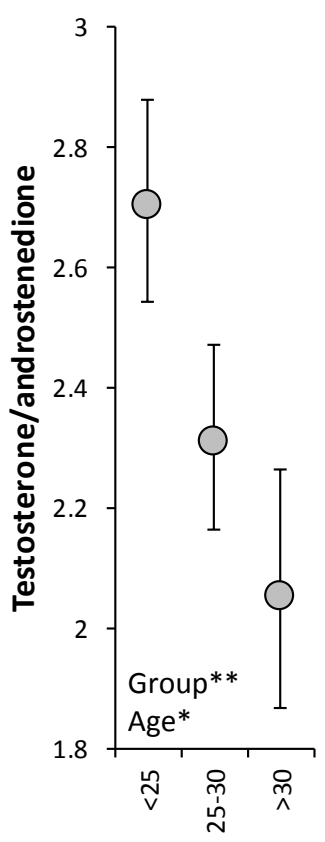

BMI $\left[\mathrm{kg} / \mathrm{m}^{2}\right]$

Figure 6: Relationships between steroid ratios reflecting the activity of type 3 17-hydroxysteroid dehydrogenase (HSD17B3) and BMI as evaluated using ANCOVA with BMI group as a main factor and age of the subject as a covariate (for details see statistical data analysis). The circles with error bars represent the group means with their $95 \%$ confidence intervals as computed for least significant difference (LSD) multiple comparisons $(p=0.05)$. The confidence, which do not overlap represent statistical significant difference between the corresponding groups. Statistical significance: NS=not significant, ${ }^{*} p<0.05$, ${ }^{* *} p<0.01,{ }^{*} p<0.001$

steroid $5 \alpha$-reducatse (SRD5A1) or $3 \beta$-hydroxysteroid dehydrogenase (HSD3B2).

These findings concur with the studies on in vitro metabolism of testosterone in the fat tissue of various localisations. In a study of intra-adipose sex steroid metabolism [9], generalized obesity (BMI) was associated with increased aromatase mRNA and $5 a$-reductase type 1 levels did not did not predict fat amount or its distribution. This supported the hypothesis that intra-adipose sex steroid metabolism is a determinant of gynoid vs. android patterns of body fat [9].

Modified androgen metabolism pathway influences fat tissue, as androgens modulate adipocyte function and affect the size of adipose tissue compartments in humans. For instance, aldo-keto reductase 1C (AKR1C) enzymes, especially AKR1C2 and AKR1C3, through local synthesis and inactivation of androgens, may be involved in the fine regulation of androgen availability in adipose tissue [10]. Type 3 $17 \beta$-hydroxysteroid dehydrogenase is co-expressed with aromatase in the abdominal preadipocytes [8].

It could be concluded that in men with increasing body mass index the formation of $\mathrm{C}_{19}$ steroids decreases from their $\mathrm{C}_{21}$ precursors, and lower $17 \beta$-hydroxysteroid dehydrogenase further confines the production of testosterone and dihydrotestosterone.

\section{Acknowledgement}

The project was supported by the grant No. NT 13542-3 of the Internal Grant Agency of the Ministry of Health IGA MZ, Czech Republic and grant GAUK No. 367511 of the Grant Agency of Charles University. 
Citation: Dušková M, Pospíšilová H, Hill M, Stárka L (2013) Obesity, Circulating Androgens and their Precursors. J Steroids Horm Sci 4: 119. doi:10.4172/2157-7536.1000119

\section{References}

1. Hofstra J, Loves S, van Wageningen B, Ruinemans-Koerts J, Jansen I, et al. (2008) High prevalence of hypogonadotropic hypogonadism in men referred for obesity treatment. Neth J Med 66: 103-109.

2. De Maddalena C, Vodo S, Petroni A, Aloisi AM (2012) Impact of testosterone on body fat composition. J Cell Physiol $227: 3744-3748$

3. Dandona P, Dhindsa S (2011) Update: Hypogonadotropic hypogonadism in type 2 diabetes and obesity. J Clin Endocrinol Metab 96: 2643-2651.

4. Allan CA, McLachlan RI (2010) Androgens and obesity. Curr Opin Endocrino Diabetes Obes 17: 224-232.

5. Wang C, Jackson G, Jones TH, Matsumoto AM, Nehra A, et al. (2011) Low testosterone associated with obesity and the metabolic syndrome contributes to sexual dysfunction and cardiovascular disease risk in men with type 2 diabetes. Diabetes Care 34: 1669-1675.

6. Glass AR (1989) Endocrine aspects of obesity. Med Clin North Am 73: 139160.

7. Cohen PG (1999) The hypogonadal-obesity cycle: role of aromatase in modulating the testosterone-estradiol shunt - a major factor in the genesis of morbid obesity. Med Hypotheses 52: 49-51.

8. Corbould AM, Bawden MJ, Lavranos TC, Rodgers RJ, Judd SJ (2002) The effect of obesity on the ratio of type $317 \beta$-hydroxysteroid dehydrogenase mRNA to cytochrome P450 aromatase mRNA in subcutaneous abdominal and intra-abdominal adipose tissue of women. Int J Obes Relat Metab Disord 26: 165-175.

9. Wake DJ, Strand M, Rask E, Westerbacka J, Livingstone DE, et al. (2007) Intra-adipose sex steroid metabolism and body fat distribution in idiopathic human obesity. Clin Endocrinol (Oxf) 66: 440-446.

10. Blouin K, Veilleux A, Luu-The V, Tchernof A (2009) Androgen metabolism in adipose tissue: recent advances. Mol Cell Endocrinol 301: 97-103.
11. Côté JA, Lessard J, Mailloux J, Laberge P, Rhéaume C, et al. (2012) Circulating $5 \alpha$-dihydrotestosterone, abdominal obesity and adipocyte characteristics in women. Horm Mol Biol Clin Invest 12: 391-400.

12. De Pergola G, Triggiani V, Giorgino F, Cospite MR, Garruti G, et al (1994) The free testosterone to dehydroepiandrosterone sulphate molar ratio as a marker of visceral fat accumulation in premenopausal obese women. Int J Obes Relat Metab Disord 18: 659-664.

13. Labrie F, Bélanger A, Cusan L, Gomez JL, Candas B (1997) Marked decline in serum concentrations of adrenal $\mathrm{C} 19$ sex steroid precursors and conjugated androgen metabolites during aging. J Clin Endocrinol Metab 82: 2396-2402.

14. Tchernof A, Labrie F (2004) Dehydroepiandrosterone, obesity and cardiovascular disease risk: a review of human studies. Eur J Endocrinol 151: $1-14$.

15. Barrett-Connor E, Ferrara A (1996) Dehydroepiandrosterone dehydroepiandrosterone sulfate, obesity, waist-hip ratio, and noninsulindependent diabetes in postmenopausal women: the Rancho Bernardo Study. J Clin Endocrinol Metab 81: 59-64.

16. Sedláčková $B$, Dušátková $L$, Zamrazilová $H$, Matucha $P$, Bičíková $M$, et al (2012) 7-oxygenated derivatives of dehydroepiandrosterone and obesity. Prague Med Rep 113: 147-155.

17. Hampl R, Putz Z, Stárka L (1990) Radioimmunolaogical determination of dihydrotestosterone and its importance for laboratory diagnostic. Biochemia Clin bohemoslov 19: 157-163.

18. Meloun M, Hill M, Militky J, Kupka K (2000) Transformation in the PC-aided biochemical data analysis. Clin Chem Lab Med 38: 553-559.

19. Meloun M, Militky J, Hill M, Brereton RG (2002) Crucial problems in regression modelling and their solutions. Analyst 127: 433-450. 